\title{
Tetramethylguanidine-functionalized silica-coated iron oxide magnetic nanoparticles catalyzed one-pot three-component synthesis of furanone derivatives
}

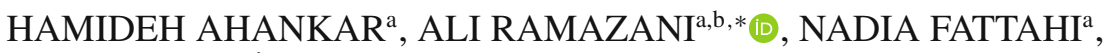 \\ KATARZYNA ŚLEPOKURA ${ }^{c}$, TADEUSZ LIS ${ }^{c}$, PEGAH AZIMZADEH ASIABI $^{\mathrm{a}}$, \\ VASYL KINZHYBALO ${ }^{\mathrm{d}}$, YOUNES HANIFEHPOUR ${ }^{\mathrm{e}, *}$ and SANG WOO JOO ${ }^{\mathrm{e}, *}$ \\ ${ }^{a}$ Department of Chemistry, University of Zanjan, P O Box 45195-313, Zanjan, Iran \\ ${ }^{b}$ Research Institute of Modern Biological Techniques, University of Zanjan, P O Box 45195-313, Zanjan, Iran \\ ${ }^{c}$ Faculty of Chemistry, University of Wrocław, 14 Joliot-Curie St, 50-383 Wrocław, Poland \\ ${ }^{\mathrm{d} I n s t i t u t e}$ of Low Temperature and Structure Research, Polish Academy of Sciences, P O Box 1410, \\ 50-950 Wrocław, Poland \\ ${ }^{\mathrm{e}}$ School of Mechanical Engineering, Yeungnam University, Gyeongsan 712-749, Republic of Korea \\ E-mail: aliramazani@znu.ac.ir; aliramazani@gmail.com; younes.hanifehpour@gmail.com; swjoo@yu.ac.kr
}

MS received 25 June 2018; revised 19 October 2018; accepted 23 October 2018; published online 26 November 2018

\begin{abstract}
Tetramethylguanidine-functionalized silica-coated iron oxide magnetic nanoparticles as a productive and reusable catalyst have been applied for the one-pot three-component synthesis of furanone derivatives (4a-0). In addition to the characterization of all products by FT-IR, ${ }^{1} \mathrm{HNMR}$ and ${ }^{13} \mathrm{CNMR}$ spectroscopy, single-crystal X-ray analysis of ethyl 5-oxo-2-phenyl-4-(phenylamino)-2,5-dihydrofuran-3carboxylate product has been made. Easy recrystallization and good-to-excellent yields (81-92\%) of furanone derivatives are notable characteristics of this procedure. The catalyst identification was performed using FT-IR, $\mathrm{XRD}, \mathrm{SEM}, \mathrm{TEM}$ and TGA techniques. It is worth noting that the nanocatalyst can be recycled with an external magnet and reused for several times.
\end{abstract}

Keywords. Furanone derivatives; three-component reaction; iron oxide; magnetic nanoparticles; tetramethylguanidine.

\section{Introduction}

During the past few decades, syntheses of important heterocycles have been extensively investigated based on multicomponent reactions (MCRs). ${ }^{1-3}$ Nowadays, three-component reactions taken into consideration in the presence of nanocatalysts, especially magnetic nanoparticles, have garnered much interest because of their unique features such as magnetic property, reusability and eco-friendliness. ${ }^{4-7}$ The tendency of magnetic nanoparticles (e.g., $\mathrm{Fe}_{3} \mathrm{O}_{4}$ ) to agglomerate makes it difficult to study their behavior. In the past few years, the surface coating or modification of iron oxide magnetic nanoparticles has emerged as a very important protocol in many applications. The main advantages of silica-coated iron oxide magnetic nanoparticles are the stability to the iron oxide magnetic nanoparticles in solution, prevention of agglomeration and possibility for linking functional groups to the surface of nanoparticles. $^{8-11}$

Butenolide skeleton has earned significant interest in organic synthesis. ${ }^{12-15}$ One class of the four-carbon heterocyclic compounds that exhibit medicinal and biological properties are the butenolides corresponding to the family of lactones. ${ }^{16}$ 2-Furanone (Figure 1), the simplest butenolide, is a usual portion of important and complex natural compounds.

As shown in Figure 2, L-ascorbic acid (1) and penicillic acid (2) are two important derivatives of the butenolide. ${ }^{17}$ Also, butenolide rings are present in a

\footnotetext{
*For correspondence

Electronic supplementary material: The online version of this article (https://doi.org/10.1007/s12039-018-1572-7) contains supplementary material, which is available to authorized users.
} 


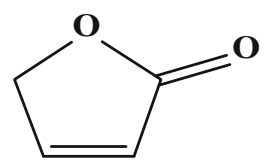

Figure 1. Chemical structure of butenolide.

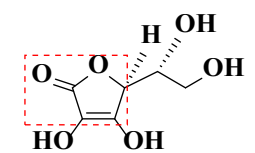

1

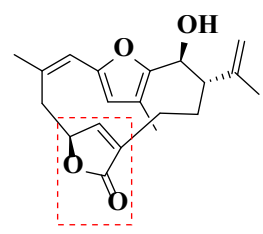

3

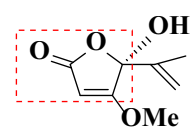

2

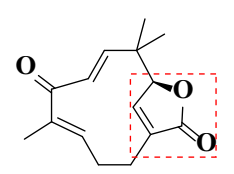

4

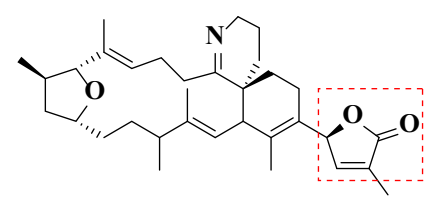

5

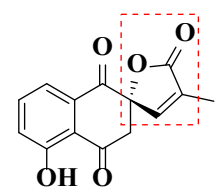

6

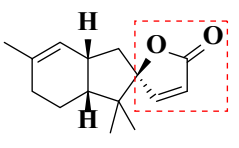

Figure 2. Chemical structures of some known butenolides.

number of natural compounds. ${ }^{18}$ Chemical structures of some known butenolides that are separated from natural compounds like bipinnatin $\mathbf{J}(\mathbf{3}),{ }^{19}$ asteriscunolide D (4) ${ }^{20}$ gymnodimine (5), ${ }^{21}$ lambertellol C (6) and spirofragilide (7) are also shown in Figure 2. ${ }^{22,23}$

Many derivatives of butenolide have shown remarkable medicinal properties such as anti-cancer, ${ }^{24}$ anti- HIV-1, ${ }^{25,26}$ anti-microbial, ${ }^{27}$ anti-fungal, ${ }^{28}$ anti-inflammatory, etc. ${ }^{29}$

In view of the importance of furanone derivatives, diverse procedures by three-component reaction have been offered for their synthesis. These include the use of $\beta$-cyclodextrin, ${ }^{30}$ tetra- $n$-butyl ammonium bisulfate, ${ }^{31}$ aluminum hydrogen sulfate, ${ }^{32}$ tin (II) chloride, ${ }^{33}$ sucrose, ${ }^{34}$ vitamin B12, ${ }^{35}$ silica sulfuric acid (SSA), ${ }^{36}$ silica gel-supported polyphosphoric acid ( $\left.\mathrm{PPA} / \mathrm{SiO}_{2}\right),{ }^{37}$ trityl chloride $(\mathrm{TrCl}),{ }^{38}$ acetic acid, ${ }^{39}$ formic acid ${ }^{40}$ and potassium hydroxide ${ }^{41}$ in solvent or solvent-free conditions at room temperature or under heat. Also, acidic ionic liquid $\left([\mathrm{Bmim}] \mathrm{HSO}_{4}\right)^{42}$ and $\left([\mathrm{H}-\mathrm{NMP}] \mathrm{HSO}_{4}\right)^{43}$ have been reported to catalyze the synthesis of furanone derivatives. Moreover, some furanone derivatives have been synthesized by green and natural catalysts such as watermelon, ${ }^{44}$ barberry ${ }^{45}$ and lemon juice. ${ }^{46}$ In recent reports, nanocatalysts have been employed to synthesize furanone derivatives, using HY Zeolite nano-powder, ${ }^{47}$ $\mathrm{ZnO}$ nanoparticles ${ }^{48}$ and sulfamic acid pyridinium chloride-functionalized $\mathrm{Fe}_{3} \mathrm{O}_{4}$ magnetic nanoparticles ${ }^{49}$ in solvent, graphene oxide nanosheet in solvent-free conditions ${ }^{50}$ and nano- $\mathrm{CdZr}_{4}\left(\mathrm{PO}_{4}\right)_{6}$ under microwave irradiation. ${ }^{51}$ It is worth mentioning, kinetics and mechanism of the reactions between para-substituted anilines, benzaldehyde and diethyl ethyne dicarboxylate for the one-pot three-component formation of furanone derivatives in formic acid has been published. ${ }^{52}$

In quest of clean and efficient synthetic methodologies, we have developed a new methodology for the synthesis of furanone derivatives by tetramethylguanidinefunctionalized silica-coated iron oxide magnetic nanoparticles $\left(\mathrm{Fe}_{3} \mathrm{O}_{4}-\mathrm{TMG}\right)$ as a reusable catalyst in a green solvent at $40^{\circ} \mathrm{C}$ (Scheme 1). Most of the above mentioned reports suffer from disadvantages such as low product yield, long reaction time, high cost, toxic organic solvent and high temperature. Furthermore, expense, difficulties in the recyclability of the catalysts and non-eco-friendly catalysts limit the usage of these methods. Easy workup, safety, the cleaner operation of the reactions, excellent yields, magnetically-retrievable and recyclable nanocatalysts are some significant features of the process, we have developed.

\section{Experimental}

\subsection{Materials and methods}

All high purity compounds and solvents were purchased from Sigma-Aldrich (Germany), Daejung (Korea), Fluka (Switzerland) and Merck (Germany) and applied without further purification. The structural properties of prepared $\mathrm{Fe}_{3} \mathrm{O}_{4}$-TMG were analyzed by FT-IR spectrometer and Xray powder diffraction (XRD) with an $X^{\prime}$ Pert-PRO advanced diffractometer using $\mathrm{Cu}(\mathrm{K} \alpha)$ radiation (wavelength: 1.5418 $\AA$ ), operated at $40 \mathrm{kV}$ and $30 \mathrm{~mA}$ at room temperature in the range of $2 \theta$ from 20 to $70^{\circ}$. The particle size and morphology of the surfaces of the sample were analyzed by scanning electron microscopy (SEM),(KYKY Co., China, Model: EM 3200). The disc was coated with gold in an ionization chamber. Transmission electron microscopy (TEM) was employed using Zeiss-EM10C TEM instrument with an accelerating voltage of $80 \mathrm{kV}$. The thermogravimetric analysis (TGA) was 


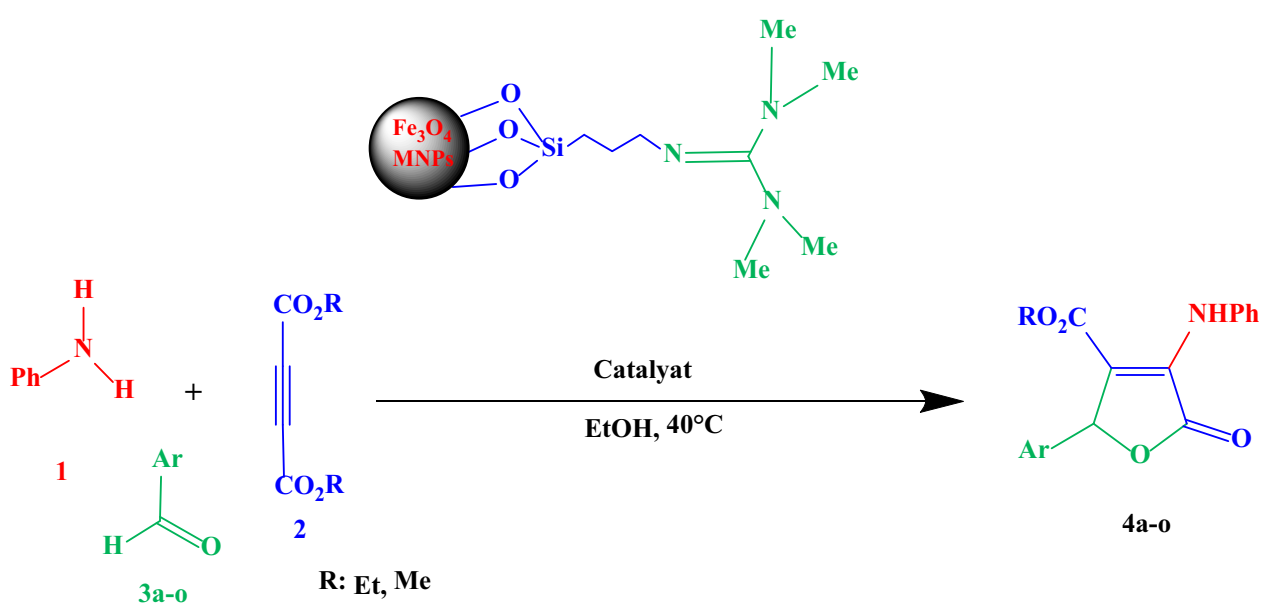

Scheme 1. Synthesis of furanone derivatives in the presence of $\mathrm{Fe}_{3} \mathrm{O}_{4}-\mathrm{TMG}$.

carried out using a Setaram-SETSYS-16/18 thermogravimetric analyzer, operated under air atmosphere, with a heating rate of $10^{\circ} \mathrm{C} / \mathrm{min}$ and in the range of $30-800^{\circ} \mathrm{C}$.

TLC methods were used to follow the reactions. Melting points were measured on an Electrothermal 9100 apparatus (LABEQUIP LTD., Markham, Ontario, Canada) and are uncorrected. ${ }^{1} \mathrm{H}$ and ${ }^{13} \mathrm{C}$ NMR spectra $\left(\mathrm{CDCl}_{3}\right)$ were recorded on a Bruker DRX-250 Avance spectrometer at 250.13 and $62.90 \mathrm{MHz}$, respectively. FT-IR spectra were measured on a Jasco 6300 FT-IR spectrometer.

\subsection{Preparation of the catalyst}

2.2a Preparation of $\mathrm{Fe}_{3} \mathrm{O}_{4}$ MNPs: $\mathrm{Fe}_{3} \mathrm{O}_{4}$ MNPs were prepared via the co-precipitation method of $\mathrm{Fe}$ (III) and Fe (II) ions (molar ratio 2:1) in ammonia solution. $\mathrm{FeCl}_{3}(980 \mathrm{mg}$ ), $\mathrm{FeSO}_{4} \cdot 7 \mathrm{H}_{2} \mathrm{O}(900 \mathrm{mg})$ were dissolved under $\mathrm{N}_{2}$ atmosphere in deionized water. The dark orange solution was stirred for 15 min at $80^{\circ} \mathrm{C}$. The $p \mathrm{H}$ value was maintained at 12 by adding ammonia solution $(28 \%)$ dropwise to the reaction mixture under constant stirring. Stirring was continued for further 30 min before cooling to room temperature. The solvent was removed by an external magnet. The particles were washed several times with water followed by ethanol to make the $\mathrm{Fe}_{3} \mathrm{O}_{4}$ MNPs free of any residual salts.

2.2b Preparation of $\mathrm{Fe}_{3} \mathrm{O}_{4} \quad \mathrm{MNPS}$ coated by (3-chloropropyl)-trimethoxysilane $\left(\mathrm{Fe}_{3} \mathrm{O}_{4}\right.$-CPTMS): $\mathrm{Fe}_{3} \mathrm{O}_{4}$ MNPs (1000 mg) was dispersed in $25 \mathrm{~mL}$ dry toluene and sonicated for one $h$ by the ultrasonic bath. The dispersion was subsequently refluxed under $\mathrm{N}_{2}$ atmosphere and stirred with the mechanical stirrer $(800 \mathrm{rpm})$ for $15 \mathrm{~min}$. Then, 3(chloropropyl) trimethoxysilane $(2 \mathrm{~mL})$ was added and the reaction mixture maintained under stirring for $24 \mathrm{~h}$. The reaction mixture was left under stir for an additional $12 \mathrm{~h}$. The solid was collected by magnetic decantation and washed with $\mathrm{EtOH}$ (6 times). The final product was dried under vacuum at room temperature for $12 \mathrm{~h}$, affording $\mathrm{Fe}_{3} \mathrm{O}_{4} @$ CPTMS. 2.2c Preparation of tetramethylguanidine-functionalized silica-coated $\mathrm{Fe}_{3} \mathrm{O}_{4} \mathrm{MNPs}\left(\mathrm{Fe}_{3} \mathrm{O}_{4} @ \mathrm{TMG}\right)$ :

The $\mathrm{Fe}_{3} \mathrm{O}_{4}$ MNPs-CPTMS (1000 mg) was dispersed in dry toluene $(20 \mathrm{~mL})$ by sonication in the ultrasonic bath for 30 min. Subsequently, tetramethylguanidine ( $4 \mathrm{mmol}, 460 \mathrm{mg}$ ) and sodium bicarbonate $(8 \mathrm{mmol}, 672 \mathrm{mg}$ ) were added and the mixture was refluxed for $28 \mathrm{~h}$. Then, the final product $\left(\mathrm{Fe}_{3} \mathrm{O}_{4} @ \mathrm{TMG}\right)$ was separated by magnetic decantation and washed twice by dry $\mathrm{CH}_{2} \mathrm{Cl}_{2}, \mathrm{C}_{2} \mathrm{H}_{5} \mathrm{OH}$ and $\mathrm{CH}_{2} \mathrm{Cl}_{2}$ respectively to remove the unattached substrates.

\subsection{General procedure for the synthesis of furanone derivatives}

A solution of aniline $\mathbf{1}(1 \mathrm{mmol})$, dialkyl ethynedicarboxylate $2(1 \mathrm{mmol})$ in ethanol $(2 \mathrm{~mL})$ was magnetically stirred at room temperature. Then, aldehyde 3a-o $(1 \mathrm{mmol})$ and tetramethylguanidine-functionalized silica-coated iron oxide magnetic nanoparticles $(80 \mathrm{mg}$ ) were added to the mixture and was stirred at $40^{\circ} \mathrm{C}$. The reaction was monitored by TLC [petroleum ether: ethyl acetate (9:2)]. After completion of the desired reaction, the resultant mixture was heated in ethanol. The catalyst was separated with an external magnet from the reaction mixture and washed with cold ethanol for reuse. The residue was concentrated under reduced pressure. The pure product was obtained by recrystallization from hot ethanol.

\subsection{Spectroscopic data of the products}

2.4a Ethyl 5-oxo-2-phenyl-4-(phenylamino)-2,5-dihydrofuran-3-carboxylate (4a) ${ }^{30}$ : M.p.: $146-149^{\circ} \mathrm{C} ;{ }^{1}$ H NMR (250.13 MHz, $\left.\mathrm{CDCl}_{3}\right): \delta(\mathrm{ppm})=8.21(\mathrm{br} \mathrm{s}, 1 \mathrm{H}$, $\mathrm{NH}), 7.19-7.57(\mathrm{~m}, 9 \mathrm{H}, \operatorname{Ar}-\mathrm{H}), 6.02(\mathrm{~s}, 1 \mathrm{H}, \mathrm{CH}), 4.08$ $\left(\mathrm{q}, J=7.00 \mathrm{~Hz}, 2 \mathrm{H}, \mathrm{CH}_{2}\right), 1.10(\mathrm{t}, J=7.00 \mathrm{~Hz}, 3 \mathrm{H}$, $\left.\mathrm{CH}_{3}\right) ;{ }^{13} \mathrm{C} \mathrm{NMR}\left(62.90 \mathrm{MHz}, \mathrm{CDCl}_{3}\right): \delta(\mathrm{ppm})=164.61$, $163.90,138.10,137.65,135.92,129.20,128.94,128.53$, 127.40, 125.21, 122.83, 114.98, 80.76, 60.67, 13.83; FT-IR $\left(\mathrm{KBr} v_{\max } / \mathrm{cm}^{-1}\right): 3314,3048,2990,1714,1686,1080$. 
2.4b Ethyl 2-(2-nitrophenyl)-5-oxo-4-(phenylamino)2,5-dihydrofuran-3-carboxylate (4b): M.p.: 140-142 ${ }^{\circ} \mathrm{C} ;{ }^{1} \mathrm{H}$ NMR $\left(250.13 \mathrm{MHz}, \mathrm{CDCl}_{3}\right): \delta(\mathrm{ppm})=8.31(\mathrm{br}$ $\mathrm{s}, 1 \mathrm{H}, \mathrm{NH}), 6.76-8.00(\mathrm{~m}, 9 \mathrm{H}, \mathrm{Ar}-\mathrm{H}), 5.73(\mathrm{~s}, 1 \mathrm{H}, \mathrm{CH}), 4.18$ $\left(\mathrm{q}, J=6.75 \mathrm{~Hz}, 2 \mathrm{H}, \mathrm{CH}_{2}\right), 0.98(\mathrm{t}, J=7.00 \mathrm{~Hz}, 3 \mathrm{H}$, $\left.\mathrm{CH}_{3}\right) ;{ }^{13} \mathrm{C} \mathrm{NMR}\left(62.90 \mathrm{MHz}, \mathrm{CDCl}_{3}\right): \delta(\mathrm{ppm})=166.14$, $163.546,149.39,138.74,13.84,137.13,133.25,130.90$, $129.89,128.78,128.11,125.65,124.90,123.22,113.95$, 73.98, 61.01; FT-IR (KBr $\left.v_{\max } / \mathrm{cm}^{-1}\right)$ : 3309, 2991, 2982, $1778,1688,1113$.

2.4c Ethyl 2-(4-nitrophenyl)-5-oxo-4-(phenylamino)2,5-dihydrofuran-3-carboxylate $(\mathbf{4 c})^{50}:$ M.p.: $144-$ $146{ }^{\circ} \mathrm{C} ;{ }^{1} \mathrm{H}$ NMR $\left(250.13 \mathrm{MHz}, \mathrm{CDCl}_{3}\right): \delta(\mathrm{ppm})=8.26$ (br s, $1 \mathrm{H}, \mathrm{NH}), 7.15-8.23(\mathrm{~m}, 9 \mathrm{H}, \mathrm{Ar}-\mathrm{H}), 6.12(\mathrm{~s}, 1 \mathrm{H}, \mathrm{CH})$, 4.00 (q, $\left.J=7.25 \mathrm{~Hz}, 2 \mathrm{H}, \mathrm{CH}_{2}\right), 1.09$ (t, $J=7.00 \mathrm{~Hz}, 3 \mathrm{H}$, $\left.\mathrm{CH}_{3}\right) ;{ }^{13} \mathrm{C} \mathrm{NMR}\left(62.90 \mathrm{MHz}, \mathrm{CDCl}_{3}\right): \delta(\mathrm{ppm})=166.05$, $163.51,148.34,143.14,138.28,137.10,128.81,128.37$, 125.76, 123.78, 123.26, 113.43, 79.11, 61.01,13.94; FT-IR $\left(\mathrm{KBr} v_{\max } / \mathrm{cm}^{-1}\right): 3423,3314,2994,2853,1784,1637$, 1199.

2.4d Ethyl 2-(4-fluorophenyl)-5-oxo-4-(phenylamino) -2,5-dihydrofuran-3-carboxylate (4d $)^{36}$ : M.p.: 141$143{ }^{\circ} \mathrm{C} ;{ }^{1} \mathrm{H}$ NMR $\left(250.13 \mathrm{MHz}, \mathrm{CDCl}_{3}\right): \delta(\mathrm{ppm})=8.19$ (br s, 1H, NH), 7.03-7.34 (m, 9H, Ar-H), $6.04(\mathrm{~s}, 1 \mathrm{H}, \mathrm{CH})$, $4.19\left(\mathrm{q}, J=6.75 \mathrm{~Hz}, 2 \mathrm{H}, \mathrm{CH}_{2}\right), 1.069(\mathrm{t}, J=7.00 \mathrm{~Hz}, 3 \mathrm{H}$, $\left.\mathrm{CH}_{3}\right) ;{ }^{13} \mathrm{C} \mathrm{NMR}\left(62.90 \mathrm{MHz}, \mathrm{CDCl}_{3}\right): \delta(\mathrm{ppm})=166.45$, $163.82,163.14\left(\mathrm{~d},{ }^{1} J_{C F}=247.95 \mathrm{~Hz}\right), 138.18,137.50$, $131.85\left(\mathrm{~d},{ }^{4} J_{C F}=3.77 \mathrm{~Hz}\right), 129.27\left(\mathrm{~d},{ }^{3} J_{C F}=8.49 \mathrm{~Hz}\right)$, $128.74,125.36,122.93,115.58\left(\mathrm{~d},{ }^{2} J_{C F}=21.83 \mathrm{~Hz}\right)$, 114.47, 60.75, 79.99, 13.86; FT-IR (KBr $\left.v_{\max } / \mathrm{cm}^{-1}\right)$ : 3321 , 2992, 2984, 1783, 1687, 1030.

2.4e Ethyl2-(2-chlorophenyl)-5-oxo-4-(phenylamino) -2,5-dihydrofuran-3-carboxylate (4e): $\quad$ M.p.: 204$207^{\circ} \mathrm{C} ;{ }^{1} \mathrm{H}$ NMR $\left(250.13 \mathrm{MHz}, \mathrm{CDCl}_{3}\right): \delta(\mathrm{ppm})=9.24$ (br s, 1H, NH), 7.16-7.44 (m, 9H, Ar-H), $6.59(\mathrm{~s}, 1 \mathrm{H}, \mathrm{CH})$, $4.08\left(\mathrm{q}, J=7.00 \mathrm{~Hz}, 2 \mathrm{H}, \mathrm{CH}_{2}\right), 1.05(\mathrm{t}, J=7.00 \mathrm{~Hz}, 3 \mathrm{H}$, $\left.\mathrm{CH}_{3}\right) ;{ }^{13} \mathrm{C}$ NMR $\left(62.90 \mathrm{MHz}, \mathrm{CDCl}_{3}\right): \delta(\mathrm{ppm})=166.48$, $163.82,138.90,137.50,133.27,130.39,130.08,128.73$, 128.51, 127.05, 125.82, 125.63, 122.96, 113.87, 77.05, 61.34, 13.80; FT-IR (KBr $\left.v_{\max } / \mathrm{cm}^{-1}\right)$ : 3314, 2921, 1778, 1693, $1499,1052$.

2.4f Ethyl 2-(4-chlorophenyl)-5-oxo-4-(phenylamino) -2,5-dihydrofuran-3-carboxylate (4f $)^{30}$ : M.p.: 158$160{ }^{\circ} \mathrm{C} ;{ }^{1} \mathrm{H}$ NMR $\left(250.13 \mathrm{MHz}, \mathrm{CDCl}_{3}\right): \delta(\mathrm{ppm})=8.19$ (br s, 1H, NH), 7.14-7.38 (m, 9H, Ar-H), 6.02 (s, 1H, CH)), $4.09\left(\mathrm{q}, J=7.00 \mathrm{~Hz}, 2 \mathrm{H}, \mathrm{CH}_{2}\right), 1.08(\mathrm{t}, J=7.00 \mathrm{~Hz}$, $3 \mathrm{H}, \mathrm{CH}_{3} ;{ }^{13} \mathrm{C} \mathrm{NMR}\left(\mathrm{CDCl}_{3}, 62.90 \mathrm{MHz}\right): \delta(\mathrm{ppm})=$ $166.41,163.74,138.15,137.47,135.08,134.58,128.78$, $128.74,125.39,122.96,114.31,79.89,60.80,13.89$; FT-IR $\left(\mathrm{KBr} v_{\max } / \mathrm{cm}^{-1}\right): 3317,2993,2982,1775,1687,1014$.

2.4g Ethyl2-(4-bromophenyl)-5-oxo-4-(phenylamino) -2,5-dihydrofuran-3-carboxylate $(\mathbf{4 g})^{46}$ : M.p.: 170-
$173{ }^{\circ} \mathrm{C} ;{ }^{1} \mathrm{H}$ NMR $\left(250.13 \mathrm{MHz}, \mathrm{CDCl}_{3}\right): \delta(\mathrm{ppm})=8.19$ (br s, 1H, NH), 7.13-7.25 (m, 9H, Arom.), 6.01 (s, 1H, CH), $4.08\left(\mathrm{q}, J=7.15 \mathrm{~Hz}, 2 \mathrm{H}, \mathrm{CH}_{2}\right), 1.07(\mathrm{t}, J=7.15 \mathrm{~Hz}, 3 \mathrm{H}$, $\left.\mathrm{CH}_{3}\right) ;{ }^{13} \mathrm{C}$ NMR $\left(62.90 \mathrm{MHz}, \mathrm{CDCl}_{3}\right): \delta(\mathrm{ppm})=166.42$, $163.73,138.12$; 137.42, 135.07, 131.74, 129.07, 128.74, 125.41, 123.27, 122.96, 114.25, 79.94, 60.83, 13.91; FT-IR $\left(\mathrm{KBr} v_{\max } / \mathrm{cm}^{-1}\right): 3316,2994,1775,1687,1030$.

2.4h Ethyl2-(naphthalen-2-yl)-5-oxo-4-(phenylamino) -2,5-dihydrofuran-3-carboxylate (4h) ${ }^{30}$ : M.p.: 178$181{ }^{\circ} \mathrm{C} ;{ }^{1} \mathrm{H}$ NMR $\left(250.13 \mathrm{MHz}, \mathrm{CDCl}_{3}\right): \delta(\mathrm{ppm})=8.24(\mathrm{br}$ s, 1H, NH), 7.18-7.87 (m, 12H, Ar-H), $6.24(\mathrm{~s}, 1 \mathrm{H}, \mathrm{CH})$, $4.05\left(\mathrm{q}, J=7.00 \mathrm{~Hz}, 2 \mathrm{H}, \mathrm{CH}_{2}\right), 1.02(\mathrm{t}, J=7.00 \mathrm{~Hz}$, $\left.3 \mathrm{H}, \mathrm{CH}_{3}\right) ;{ }^{13} \mathrm{C} \mathrm{NMR}\left(62.90 \mathrm{MHz}, \mathrm{CDCl}_{3}\right): \delta(\mathrm{ppm})=$ $166.73,163.95,137.63,138.17,133.66,133.18,133.08$, $128.76,128.50,128.09,127.77,127.59,126.67,126.46$, $125.27,123.98,122.89,114.97,80.93,60.72,13.86$; FTIR $\left(\mathrm{KBr} v_{\max } / \mathrm{cm}^{-1}\right)$ : 3309, 3046, 2992, 2970, 1775, 1687, 1080 .

2.4i Eethyl 5-oxo-4-(phenylamino)-2-(p-tolyl)-2, 5-dihydrofuran-3-carboxylate $(\mathbf{4 i})^{30}$ : M.p.: $157-$ $162{ }^{\circ} \mathrm{C} ;{ }^{1} \mathrm{H}$ NMR $\left(250.13 \mathrm{MHz}, \mathrm{CDCl}_{3}\right): \delta(\mathrm{ppm})=8.17$ (br s, 1H, NH), 7.15-7.37 (m, 9H, Ar-H), $6.04(\mathrm{~s}, 1 \mathrm{H}, \mathrm{CH})$, $4.08\left(\mathrm{q}, J=7.13 \mathrm{~Hz}, 2 \mathrm{H}, \mathrm{CH}_{2}\right), 1.07(\mathrm{t}, J=7.13 \mathrm{~Hz}$, $\left.3 \mathrm{H}, \mathrm{CH}_{3}\right), 2.37(\mathrm{~s}, 3 \mathrm{H}) ;{ }^{13} \mathrm{C}$ NMR $\left(62.90 \mathrm{MHz}, \mathrm{CDCl}_{3}\right)$ : $\delta(\mathrm{ppm})=166.82,163.93,139.14,137.94,137.77,132.90$, $129.25,128.70,127.34,125.12,122.75,115.14,80.76,60.69$, 21.28, 13.88; FT-IR (KBr $\left.v_{\max } / \mathrm{cm}^{-1}\right)$ : 3316, 2993, 1775, $1688,1029$.

2.4j Ethyl 2-(4-(methylthio) phenyl)-5-oxo-4(phenylamino)-2,5-dihydrofuran-3-carboxylate $(\mathbf{4 j})$ : M.p.: $158-160{ }^{\circ} \mathrm{C} ;{ }^{1} \mathrm{H}$ NMR $\left(250.13 \mathrm{MHz}, \mathrm{CDCl}_{3}\right): \delta(\mathrm{ppm})$ $=8.19(\mathrm{br} \mathrm{s}, 1 \mathrm{H}, \mathrm{NH}), 7.06-7.51(\mathrm{~m}, 9 \mathrm{H}, \mathrm{Ar}-\mathrm{H}), 6.04(\mathrm{~s}, 1 \mathrm{H}$, $\mathrm{CH}), 4.09$ (q, $\left.J=7.00 \mathrm{~Hz}, 2 \mathrm{H}, \mathrm{CH}_{2}\right), 2.49$ (s, 3H), 1.07 (t, $\left.J=6.75 \mathrm{~Hz}, 3 \mathrm{H}, \mathrm{CH}_{3}\right) ;{ }^{13} \mathrm{C} \mathrm{NMR}\left(62.90 \mathrm{MHz}, \mathrm{CDCl}_{3}\right)$ : $\delta(\mathrm{ppm})=166.62,163.84,140.02,138.05,137.64,132.53$, $128.71,127.84,126.23,125.23,122.82,114.70,80.41,60.72$, 15.53, 13.89; FT-IR (KBr $\left.v_{\max } / \mathrm{cm}^{-1}\right)$ : 3309, 2992, 2922, 1773, 1686, 1033.

2.4k Methyl 5-oxo-2-phenyl-4-(phenylamino)-2, 5-dihydrofuran-3-carboxylate $\quad(\mathbf{4 k})^{33}$ : $\quad$ M.p.: $160-$ $163^{\circ} \mathrm{C} ;{ }^{1}{ }^{1} \mathrm{H}$ NMR $\left(250.13 \mathrm{MHz}, \mathrm{CDCl}_{3}\right): \delta(\mathrm{ppm})=8.20$ (br s, 1H, NH), 7.16-7.38 (m, 10H, Ar-H), $6.06(\mathrm{~s}, 1 \mathrm{H}$, $\mathrm{CH}), 3.62\left(\mathrm{~s}, 3 \mathrm{H}, \mathrm{CH}_{3}\right) ;{ }^{13} \mathrm{C} \mathrm{NMR}\left(62.90 \mathrm{MHz}, \mathrm{CDCl}_{3}\right)$ : $\delta(\mathrm{ppm})=166.49,164.28,138.39,137.55,135.90,129.28$, 128.72, 128.65, 127.37, 125.35, 123.03, 114.36, 80.70, 51.61; FT-IR $\left(\mathrm{KBr} v_{\max } / \mathrm{cm}^{-1}\right): 3313,3028,2952,1774,1676$, 1077.

2.41 Methyl 2-(3-chlorophenyl)-5-oxo-4-(phenylamino)-2,5-dihydrofuran-3-carboxylate (4l): M.p.: 132$134{ }^{\circ} \mathrm{C} ;{ }^{1} \mathrm{H}$ NMR $\left(250.13 \mathrm{MHz}, \mathrm{CDCl}_{3}\right): \delta(\mathrm{ppm})=8.23$ (br s, 1H, NH), 7.16-7.39 (m, 9H, Ar-H), 6.01 (s, 1H, $\mathrm{CH}), 3.65\left(\mathrm{~s}, 3 \mathrm{H}, \mathrm{CH}_{3}\right) ;{ }^{13} \mathrm{C} \mathrm{NMR}\left(62.90 \mathrm{MHz}, \mathrm{CDCl}_{3}\right)$ : 


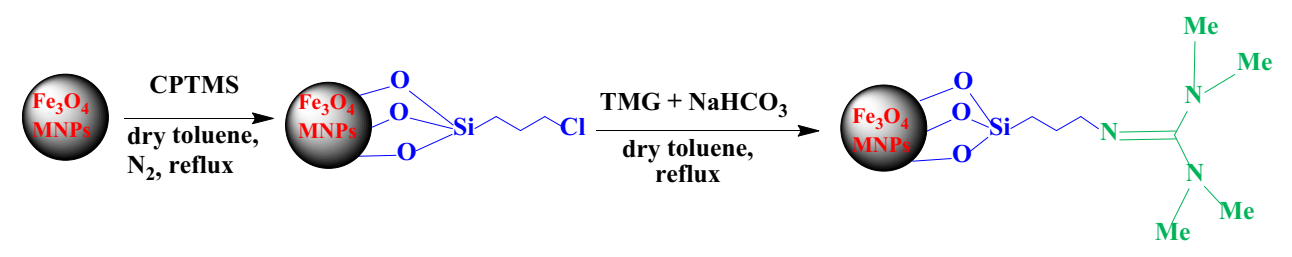

Scheme 2. The preparation of $\mathrm{Fe}_{3} \mathrm{O}_{4}$-TMG.

$\delta(\mathrm{ppm})=166.11,164.09,138.53,138.02,137.29,134.53$, $129.93,129.46,128.74,127.47,125.63,125.59,123.24$, 113.51, 79.76, 51.70; FT-IR (KBr $\left.v_{\max } / \mathrm{cm}^{-1}\right)$ : 3316, 3051, 2960, 1788, 1692, 1027.

2.4m Methyl2-(4-chlorophenyl)-5-oxo-4-(phenylamino)-2,5-dihydrofuran-3-carboxylate $(\mathbf{4 m})^{33}$ : M.p.: 151-153 ${ }^{\circ} \mathrm{C} ;{ }^{1} \mathrm{H}$ NMR $\left(250.13 \mathrm{MHz}, \mathrm{CDCl}_{3}\right): \delta(\mathrm{ppm})=$ 8.23 (br s, 1H, NH), 7.14-7.38 (m, 9H, Ar-H), $6.02(\mathrm{~s}, 1 \mathrm{H}$, $\mathrm{CH}), 3.63\left(\mathrm{~s}, 3 \mathrm{H}, \mathrm{CH}_{3}\right) ;{ }^{13} \mathrm{C} \mathrm{NMR}\left(62.90 \mathrm{MHz}, \mathrm{CDCl}_{3}\right)$ : $\delta(\mathrm{ppm})=166.27,164.12,138.42,137.35,135.15,128.90$, 128.79, 124.51, 125.54, 123.16, 123.08, 113.75, 79.81, 51.71; FT-IR $\left(\mathrm{KBr} v_{\max } / \mathrm{cm}^{-1}\right)$ : 3316, 3051, 2960, 1788, 1692, 1027.

2.4n Methyl2-(4-bromophenyl)-5-oxo-4-(phenylamino)-2,5-dihydrofuran-3-carboxylate (4n) ${ }^{51}$ : M.p.: $164-$ $166{ }^{\circ} \mathrm{C} ;{ }^{1} \mathrm{H}$ NMR $\left(250.13 \mathrm{MHz}, \mathrm{CDCl}_{3}\right): \delta(\mathrm{ppm})=8.20$ (br s, 1H, NH), 7.14-7.53 (m, 9H, Ar-H), $6.01(\mathrm{~s}, 1 \mathrm{H}$, $\mathrm{CH}), 3.62\left(\mathrm{~s}, 3 \mathrm{H}, \mathrm{CH}_{3}\right) ;{ }^{13} \mathrm{C} \mathrm{NMR}\left(62.90 \mathrm{MHz}, \mathrm{CDCl}_{3}\right)$ : $\delta(\mathrm{ppm})=166.25,164.11,138.41,137.32,135.02,131.85$, 129.05, 125.55, 123.36, 123.36, 122.06, 113.68, 51.72, 79.88; FT-IR $\left(\mathrm{KBr} v_{\max } / \mathrm{cm}^{-1}\right)$ : 3321, 3051, 2956, 1778, 1693, 1028.

2.4o Methyl2-(naphthalen-2-yl)-5-oxo-4-(phenylamino)-2,5-dihydrofuran-3-carboxylate (4o): M.p.: 176$178^{\circ} \mathrm{C} ;{ }^{1} \mathrm{H}$ NMR $\left(250.13 \mathrm{MHz}, \mathrm{CDCl}_{3}\right): \delta(\mathrm{ppm})=8.30(\mathrm{br}$ $\mathrm{s}, 1 \mathrm{H}, \mathrm{NH}), 7.23-7.88(\mathrm{~m}, 12 \mathrm{H}, \mathrm{Ar}-\mathrm{H}), 6.24(\mathrm{~s}, 1 \mathrm{H}, \mathrm{CH}), 3.59$ $\left(\mathrm{s}, 3 \mathrm{H}, \mathrm{CH}_{3}\right) ;{ }^{13} \mathrm{C} \mathrm{NMR}\left(62.90 \mathrm{MHz}, \mathrm{CDCl}_{3}\right): \delta(\mathrm{ppm})=$ $166.22,164.39,138.54,137.51,133.72,133.31,128.77$, $128.66,128.21,127.81,127.65,126.77,126.52,125.43$, 124.01, $\quad 123.10, \quad 114.33, \quad 80.93, \quad 51.75 ; \quad$ FT-IR $\left(\mathrm{KBr} v_{\max } / \mathrm{cm}^{-1}\right): 3314,3048,2956,1781,1640,1027$.

\section{$2.5 X$-ray crystallography}

Synthesized pure powder of ethyl 5-oxo-2-phenyl4-(phenylamino)-2,5-dihydrofuran-3-carboxylate (4a) was solved in hot ethanol. X-ray quality crystals of $\mathbf{4 a}$ were obtained in excellent yield after gradual evaporation of the mother liquor at room temperature.

The crystallographic measurement of $\mathbf{4 a}$ was carried out on a Kuma KM4-CCD к-geometry automated four-circle diffractometer equipped with a CCD Saphire2 camera, respectively, and graphite-monochromatized $\operatorname{Mo} K \alpha$ radiation $(\lambda=$

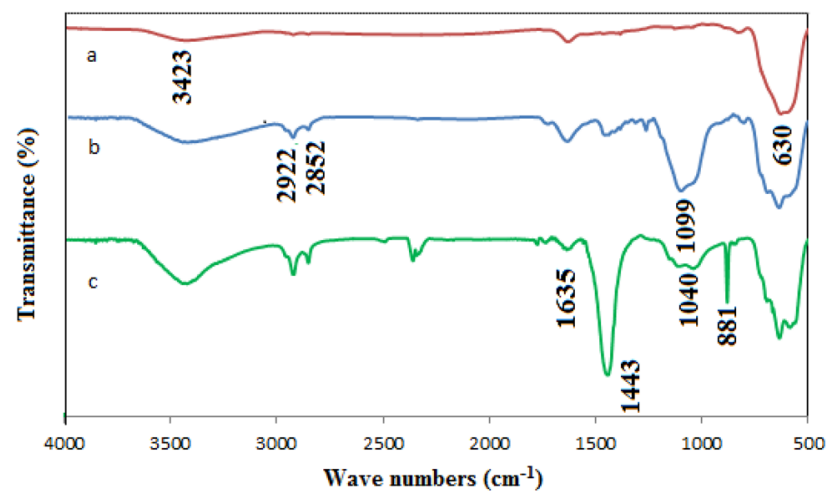

Figure 3. FT-IR spectra of (a) $\mathrm{Fe}_{3} \mathrm{O}_{4}$ MNPs, (b) $\mathrm{Fe}_{3} \mathrm{O}_{4}-\mathrm{CPTMS}$ and (c) $\mathrm{Fe}_{3} \mathrm{O}_{4}-\mathrm{TMG}$.

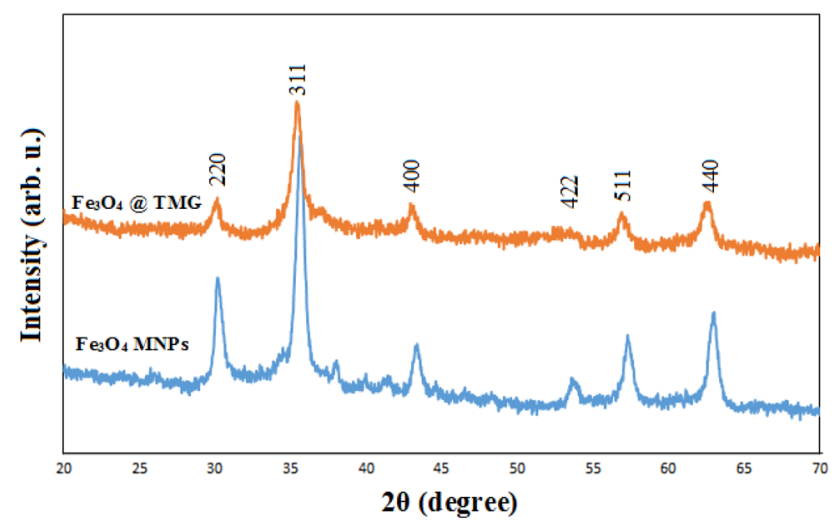

Figure 4. XRD patterns of (a) $\mathrm{Fe}_{3} \mathrm{O}_{4}$ MNPs and (b) $\mathrm{Fe}_{3} \mathrm{O}_{4}-\mathrm{TMG}$.

$0.71073 \AA$ ). The data were gathered at 100 (2) by using the Oxford-Cryosystems cooler. Data were corrected for Lorentz and polarization effects. Data collection, cell refinement, data reduction and analysis were done with CrysAlis CCD and CrysAlisRED, respectively. ${ }^{53}$ The structure was solved by direct methods with the SHELXS97 program, ${ }^{54}$ and refined by a full-matrix least-squares technique with SHELXL2014 ${ }^{55}$ and anisotropic thermal parameters for all non- $\mathrm{H}$ atoms. $\mathrm{H}$ atoms were found in difference Fourier maps. In the final refinement cycles, the $\mathrm{C}$-bonded $\mathrm{H}$ atoms were repositioned in their calculated positions and refined using a riding model, with $\mathrm{C}-\mathrm{H}=0.95-1.00 \AA$, and with $U_{\text {iso }}(\mathrm{H})=1.2 U_{\text {eq }}(\mathrm{C})$ for $\mathrm{CH}, \mathrm{CH}_{2}$ and $1.5 U_{\text {eq }}(\mathrm{C})$ for $\mathrm{CH}_{3}$. N-bonded $\mathrm{H}$ atom was refined freely. Figures were made with the Diamond 

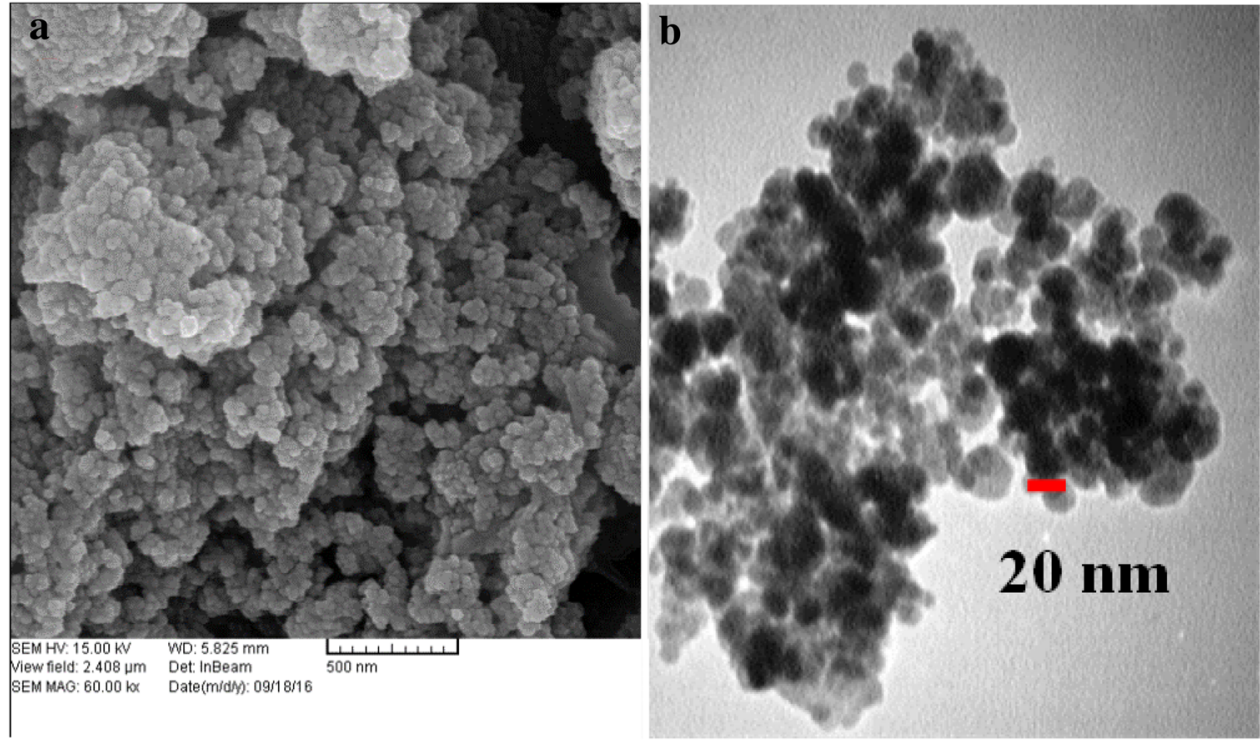

Figure 5. (a) SEM and (b) TEM images of $\mathrm{Fe}_{3} \mathrm{O}_{4}$-TMG.

program. ${ }^{56}$ Details of the conditions for the data collection and the structure refinement are given in the crystallographic information file (CIF) deposited with The Cambridge Crystallographic Data Centre (www.ccdc.cam.ac.uk/; deposition number CCDC-1468805) and provided as Supplementary Information.

Crystal data for 4a: $\mathrm{C}_{19} \mathrm{H}_{17} \mathrm{NO}_{4}, M w=323.33$, yellowish block, crystal size $0.55 \times 0.42 \times 0.33 \mathrm{~mm}$, orthorhombic, space group $\mathrm{Pbca}, a=16.317(5), b=7.390(2), c=$ 25.973(8) $\mathrm{A}, V=3131.9(16) \AA^{3}, T=100(2) \mathrm{K}, Z=8$, $\mu=0.10 \mathrm{~mm}^{-1}$ (for $\operatorname{Mo} K \alpha, \lambda=0.71073 \AA$ ), multi-scan absorption correction, $T_{\min }=0.765, T_{\max }=1.000,25010$ reflections measured, 5116 unique $(R$ int $=0.054), 3732$ observed $(I>2 \sigma(I)),(\sin \theta / \lambda)_{\max } 0.840 \AA^{-1}, 222$ parameters, 0 restraints, $R=0.055, w R=0.142$ (observed refl.), GOOF $=S=1.00,\left(\Delta \rho_{\max }\right)=0.50$ and $\left(\Delta \rho_{\min }\right)=$ $-0.27 \mathrm{e} \cdot \AA^{-3}$.

\section{Results and Discussion}

\subsection{Characterization of catalyst}

Tetramethylguanidine-functionalized silica-coated iron oxide magnetic nanoparticles $\left(\mathrm{Fe}_{3} \mathrm{O}_{4}\right.$-TMG) as a catalyst was prepared by the indicated summary path in Scheme 2. The detailed description of the catalyst preparation is given in preparation section of catalyst.

The catalyst was characterized using a variety of different techniques. The FT-IR spectrum of the $\mathrm{Fe}_{3} \mathrm{O}_{4}$ MNPs is demonstrated in Figure 3a. The principle absorption band at $630 \mathrm{~cm}^{-1}$ corresponded to the characteristic vibration absorption peak of $\mathrm{Fe}-\mathrm{O}$ bond.
Table 1. The effect of the solvents on the synthesis of furanone derivatives.

\begin{tabular}{lccc}
\hline Entry & Solvent & Time (h) & Yield $(\%)^{\mathrm{a}}$ \\
\hline 1 & Water & 12 & 32 \\
2 & Water-Ethanol & 12 & 82 \\
3 & Ethanol & 12 & 91 \\
4 & Methanol & 12 & 74 \\
5 & Water-acetonitrile & 12 & 69 \\
6 & Acetonitrile & 12 & 75 \\
\hline
\end{tabular}

Reaction conditions: aniline $\mathbf{1}$ (1mmol), diethyl ethynedicarboxylate $\mathbf{2}$ (1 $\mathrm{mmol})$, 4chlorobenzaldehyde 3f (1 mmol), tetramethylguanidine-functionalized silica-coated iron oxide magnetic nanoparticles $(80 \mathrm{mg})$ in different solvents $(2 \mathrm{~mL})$ at $40^{\circ} \mathrm{C}$.

${ }^{\text {a }}$ Isolated yield.

Moreover, a broad absorption band is present at $3423 \mathrm{~cm}^{-1}$ for stretching mode of the large number of $\mathrm{OH}$ groups.

$\mathrm{Fe}_{3} \mathrm{O}_{4}$ MNPs coated by (3-chloropropyl)-trime thoxysilane $\left(\mathrm{Fe}_{3} \mathrm{O}_{4} @ \mathrm{CPTMS}\right)$ is confirmed by the bands at 2922, 2852 and $1099 \mathrm{~cm}^{-1}$ assigned $\mathrm{C}-\mathrm{H}$ stretching vibrations and $\mathrm{Fe}-\mathrm{O}-\mathrm{Si}$, respectively in Figure $3 \mathrm{~b}$. In addition, tetramethylguanidine-functionalized silica-coated iron oxide magnetic nanoparticle $\left(\mathrm{Fe}_{3} \mathrm{O}_{4} @ \mathrm{TMG}\right)$ is affirmed with 1443 and $1635 \mathrm{~cm}^{-1}$ bands corresponding to the $\mathrm{C}-\mathrm{N}$ and $\mathrm{C}=\mathrm{N}$ stretch respectively are shown in Figure 3c.

The X-ray powder diffraction (XRD) measurements uncoated $\mathrm{Fe}_{3} \mathrm{O}_{4}$ MNPs and coated $\mathrm{Fe}_{3} \mathrm{O}_{4}$-TMG were 


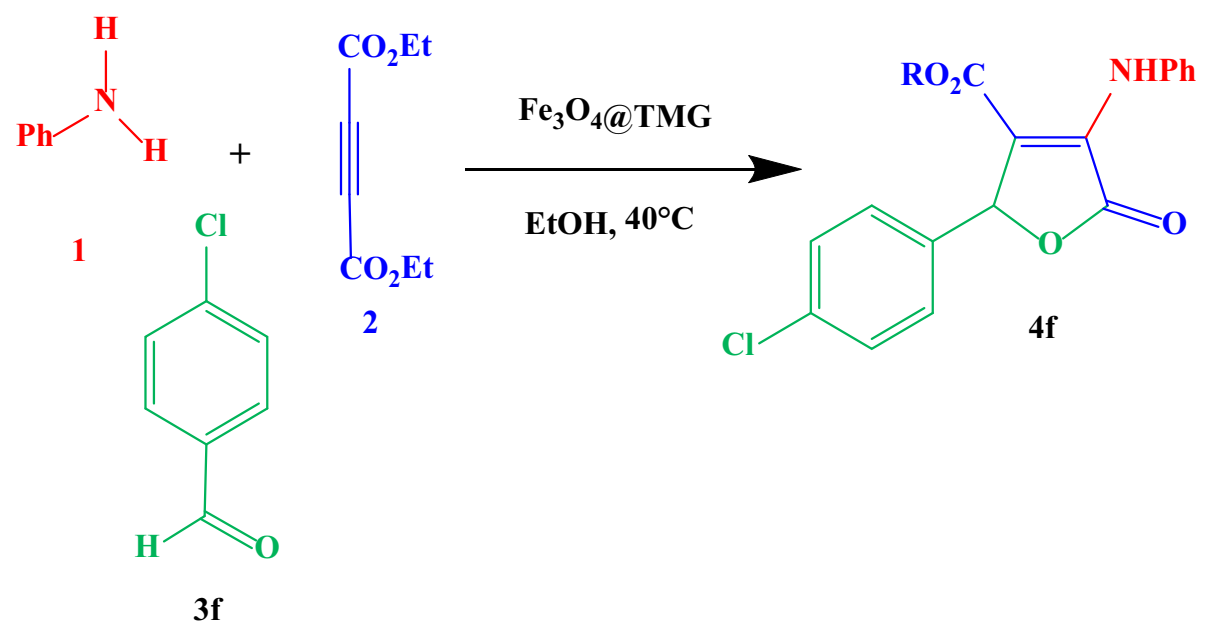

Scheme 3. Synthesis of $\mathbf{4} \mathbf{f}$ in the presence of $\mathrm{Fe}_{3} \mathrm{O}_{4}-\mathrm{TMG}$.

determined by XRD analysis (Figure 4). The results indicate that the cubic spinel structure of $\mathrm{Fe}_{3} \mathrm{O}_{4}$ MNPs (JSPDS Card no. 01-075-0449). Observed Miller indices in (2 200$),\left(\begin{array}{lll}3 & 1 & 1\end{array}\right),\left(\begin{array}{lll}4 & 0 & 0\end{array}\right),\left(\begin{array}{lll}4 & 2 & 2\end{array}\right),\left(\begin{array}{lll}5 & 1 & 1\end{array}\right)$, and (4 $40)$ planes is related to a series of diffraction peaks at $2 \theta=30.66^{\circ}, 35.78^{\circ}, 43.53^{\circ}, 53.47^{\circ}, 57.83^{\circ}$ and $63.25^{\circ}$, respectively. The average diameter of the $\mathrm{Fe}_{3} \mathrm{O}_{4}$-TMG nanoparticles was characterized to be $20 \mathrm{~nm}$ by the well-known Debye-Scherrer equation via XRD data $(\mathrm{D}=0.94 \lambda / \mathrm{B} \cos \theta)$ according to the broadening of the $\left(\begin{array}{lll}3 & 1 & 1\end{array}\right)$ characteristic peak of the XRD pattern. The results show prosperous grafting of tetramethylguanidinone to magnetic nanoparticles (Figure 4). Although peak height has decreased because of coating, the cubic spinel structure of $\mathrm{Fe}_{3} \mathrm{O}_{4}$ MNPs has been preserved during grafting.

The scanning electron microscopy (SEM) image demonstrated external morphology and particle size and of the $\mathrm{Fe}_{3} \mathrm{O}_{4}$-TMG powder as a catalyst (Figure 5a). It can be seen from the SEM image, the size of the grains of nanoparticles with spherical morphology is $18-25 \mathrm{~nm}$ that is according with the XRD pattern. The morphology and particles size of the Fe3O4-TMG was investigated by transmission electron microscopy (TEM) image (Figure 5b). This image has revealed more accurate information on the grains measure and morphology of nanocatalyst. The mean size of the spherical particles was $20 \mathrm{~nm}$ which is fully consistent with the sizes of the XRD pattern and SEM image.

The thermogravimetric analysis (TGA) curves illustrate the decomposition of the $\mathrm{Fe}_{3} \mathrm{O}_{4}$-CPTMS and $\mathrm{Fe}_{3} \mathrm{O}_{4}$-TMG. In both curves, the first step at $25-150{ }^{\circ} \mathrm{C}$ is ascribed to the elimination of physical and chemical absorption of water. In the next step, the weight loss of organic groups (3-chloropropyl)-trimethoxysilane
Table 2. The effect of temperature on the synthesis of furanone derivatives.

\begin{tabular}{|c|c|c|c|}
\hline Entry & Temperature & Time $(\mathrm{h})$ & Yield $(\%)^{\mathrm{a}}$ \\
\hline 1 & Room temperature & 12 & 75 \\
\hline 2 & 40 & 12 & 91 \\
\hline 3 & 60 & 12 & 91 \\
\hline 4 & 80 & 12 & 88 \\
\hline
\end{tabular}
diethyl ethynedicarboxylate 2 (1 mmol), 4-chlorobenzaldehyde 3f (1 mmol), tetramethylguanidine-functionalized silica-coated iron oxide magnetic nanoparticles $(80 \mathrm{mg})$ in ethanol solvent $(2$ $\mathrm{mL})$ at different temperature.

${ }^{\mathrm{a}}$ Isolated yield.

Table 3. The effect of the catalyst amount on the synthesis of furanone derivatives.

\begin{tabular}{|c|c|c|c|}
\hline Entry & Catalyst (mg) & Time (h) & Yield $(\%)^{\mathrm{a}}$ \\
\hline 1 & - & 12 & 38 \\
\hline 2 & 25 & 12 & 66 \\
\hline 3 & 50 & 12 & 75 \\
\hline 4 & 80 & 12 & 91 \\
\hline \multicolumn{4}{|c|}{$\begin{array}{l}\text { Reaction conditions: aniline } \mathbf{1} \text { ( } 1 \mathrm{mmol}) \text {, } \\
\text { diethyl ethynedicarboxylate } \mathbf{2}(1 \mathrm{mmol}) \text {, } \\
\text { 4-chlorobenzaldehyde } \mathbf{3 f} \text { (1 } \mathrm{mmol}) \text {, } \\
\text { various amounts of tetramethylguanidine- } \\
\text { functionalized silica-coated iron oxide } \\
\text { magnetic nanoparticles in ethanol solvent } \\
(2 \mathrm{~mL}) \text { at } 40^{\circ} \mathrm{C} \text {. } \\
\text { a Isolated yield. }\end{array}$} \\
\hline
\end{tabular}

(CPTMS) and tetramethylguanidine (TMG) was calculated $3.3 \%$ and $14.36 \%$ at $200-800{ }^{\circ} \mathrm{C}$, respectively (Figures S46 and S47 in Supplementary Information). 
Table 4. Tetramethylguanidine-functionalized silica-coated iron oxide magnetic nanoparticles catalyzed synthesis of furanone derivatives.

\begin{tabular}{|c|c|c|c|c|c|}
\hline Entry & $\mathrm{Ar}$ & $\mathrm{R}$ & Product & Time (h) & Yield $(\%)^{\mathrm{a}}$ \\
\hline 1 & $\mathrm{Ph}$ & Et & $4 a$ & 12 & 85 \\
\hline 2 & $2-\mathrm{NO}_{2}-\mathrm{C}_{6} \mathrm{H}_{4}$ & Et & $4 b$ & 12 & 82 \\
\hline 3 & $4-\mathrm{NO}_{2}-\mathrm{C}_{6} \mathrm{H}_{4}$ & $\mathrm{Et}$ & $4 c$ & 10 & 89 \\
\hline 4 & $4-\mathrm{F}-\mathrm{C}_{6} \mathrm{H}_{4}$ & $\mathrm{Et}$ & $4 d$ & 10 & 91 \\
\hline 5 & $2-\mathrm{Cl}-\mathrm{C}_{6} \mathrm{H}_{4}$ & Et & $4 e$ & 12 & 90 \\
\hline 6 & $4-\mathrm{Cl}-\mathrm{C}_{6} \mathrm{H}_{4}$ & Et & $\mathbf{4 f}$ & 12 & 91 \\
\hline 7 & $4-\mathrm{Br}-\mathrm{C}_{6} \mathrm{H}_{4}$ & Et & $4 g$ & 12 & 85 \\
\hline
\end{tabular}


Table 4. (contd.)

\begin{tabular}{|c|c|c|c|c|c|}
\hline Entry & $\mathrm{Ar}$ & $\mathrm{R}$ & Product & Time (h) & Yield $(\%)^{\mathrm{a}}$ \\
\hline 8 & 2-Naphthal & Et & & 12 & 90 \\
\hline 9 & 4-Me- $\mathrm{C}_{6} \mathrm{H}_{4}$ & Et & & 13 & 92 \\
\hline 10 & $4-\mathrm{MeS}-\mathrm{C}_{6} \mathrm{H}_{4}$ & Et & & 11 & 89 \\
\hline 11 & $\mathrm{Ph}$ & $\mathrm{Me}$ & & 12 & 91 \\
\hline 12 & $3-\mathrm{Cl}-\mathrm{C}_{6} \mathrm{H}_{4}$ & $\mathrm{Me}$ & & 13 & 87 \\
\hline & & & 41 & & \\
\hline 13 & $4-\mathrm{Cl}-\mathrm{C}_{6} \mathrm{H}_{4}$ & $\mathrm{Me}$ & & 12 & 89 \\
\hline 14 & $4-\mathrm{Br}-\mathrm{C}_{6} \mathrm{H}_{4}$ & $\mathrm{Me}$ & & 12 & 87 \\
\hline 15 & 2-Naphthal & $\mathrm{Me}$ & & 12 & 89 \\
\hline
\end{tabular}

Reaction conditions: aniline $\mathbf{1}(1 \mathrm{mmol})$, dialkylethynedicarboxylate $\mathbf{2}(1 \mathrm{mmol})$, aldehyde 3a-o $(1 \mathrm{mmol})$, tetramethylguanidine-functionalized silica-coated iron oxide magnetic nanoparticles $(80 \mathrm{mg})$ in ethanol solvent $(2 \mathrm{~mL})$ at $40^{\circ} \mathrm{C}$.

${ }^{\mathrm{b}}$ Isolated yield. 


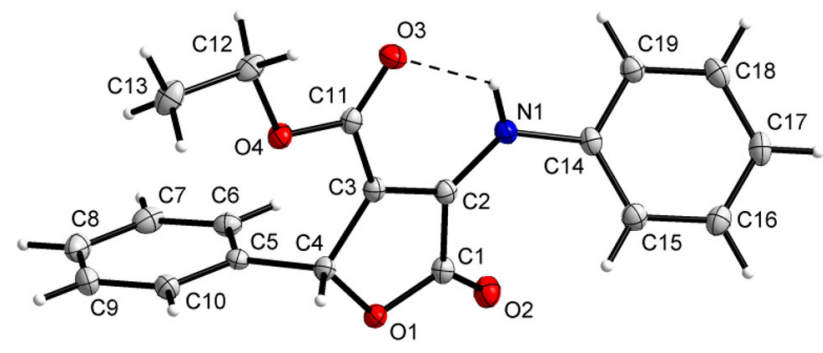

Figure 6. X-ray structure of $(\boldsymbol{R})-\mathbf{4 a}$, showing the atom-numbering scheme and the symmetry-independent hydrogen bond (dashed line). Displacement ellipsoids are drawn at the $50 \%$ probability level.

\subsection{Application of $\mathrm{Fe}_{3} \mathrm{O}_{4}-\mathrm{TMG}$ in the synthesis of furanone derivatives}

To survey the appropriate conditions for the synthesis of furanone derivatives $(\mathbf{4 a - 0})$, various reaction conditions have been checked. We optimized the reaction conditions such as effects of solvent, temperature and catalyst amount.

To begin, different solvents listed in Table 1 were investigated in the synthesis of $\mathbf{4} \mathbf{f}$ as a sample compound (Scheme 3). The foremost option was selected by the reaction of aniline 1, diethyl acetylenedicarboxylate 2,4-chlorobenzaldehyde $\mathbf{3 f}$ and tetramethylguanidinefunctionalized silica-coated iron oxide magnetic nanoparticles as a catalyst in ethanol solvent at $40^{\circ} \mathrm{C}$ to produce $\mathbf{4 f}$ in $12 \mathrm{~h}$ with $91 \%$ yield (Table 1, entry 3 ). Other solvents are in (Table 1, entries 1, 2 and 4-6) afforded low to moderate yields of the desired products.

It may be mentioned that we tested some reactions (Scheme 3) at room temperature 40,60 and $80^{\circ} \mathrm{C}$ in $12 \mathrm{~h}$ (Table 2). At room temperature, the mixture of starting materials was seen during the reaction. After increasing the temperature to $40^{\circ} \mathrm{C}$, after $12 \mathrm{~h}$ the reaction was completed with $91 \%$ yield (Table 2, entry 2). Raising the temperature to $60^{\circ} \mathrm{C}$ did not have any great impact on the yield of the reaction. Of course, increasing the temperature to $80^{\circ} \mathrm{C}$ has caused reducing the main product. Therefore, the temperature, $40^{\circ} \mathrm{C}$ was chosen as the appropriate temperature for the reactions.
To find out the optimized amount of the magnetic nanoparticles supported tetramethylguanidine catalyst for the synthesis of compound $\mathbf{4 f}$ (Table 4, entry 6) was carried out by varying the quantity of catalyst, as shown below (Table 3, entries 2-4). The maximum yield of the compound $\mathbf{4 f}$ was seen when $80 \mathrm{mg}$ of catalyst was applied.

After detecting more efficient and green solvent, the desired temperature and amount of catalyst, all reactions were examined with optimized values conforming to Scheme 1. Thereafter, aromatic aldehydes (3a-0) containing different groups on the aromatic ring at ortho, meta, and para positions were appraised. As shown in (Table 4), the reaction times lasted from 10 to $13 \mathrm{~h}$ and yields of the isolated products were good-to-excellent (81-92\%). Some aldehydes such as trans-cinnamaldehyde, salicylaldehyde, 5-bromosalicyaldehyde, 4-(dimethylamino) benzaldehyde, acetaldehyde, 2-hydroxy-1-naphthaldehyde, pyrrole-2-carboxaldehyde, thiophene-2-carboxaldehyde, furfural, 3- phenylpropionaldehyde, isobutyraldehyde, formaldehyde and glyoxal under the reaction conditions were tested, but desired product could not be produced and the mixture of starting materials and another product was seen (based on TLC investigation). 3pyridinecarboxaldehyde, 4-pyridinecarboxaldehyde and 4-hydroxybenzaldehyde had low yield under the same conditions.

Eventually, the reusability and catalytic activity of $\mathrm{Fe}_{3} \mathrm{O}_{4}$-TMG under the model reaction for the synthesis of compound $\mathbf{4 f}$ (Table 4, entry 6) were considered. Every time, the catalyst was separated with an external magnet from the reaction mixture, rinsed with ethanol, dried at room temperature to remove any remaining ethanol and reused in the subsequent reactions. The yields for five consecutive runs were 91, 90, 91, 89 and $92 \%$, respectively. The results showed that the catalyst could be reused for five times without any excessive reduction in its function.

As far as we know, this procedure is the most efficient, clean and convenient procedure, and is applicable

Table 5. Hydrogen-bond and $\mathrm{C}-\mathrm{H} \cdots \pi$ contacts geometry $\left(\AA,{ }^{\circ}\right)$ for $\mathbf{4 a}$.

\begin{tabular}{lcccc}
\hline$D-\mathrm{H} \cdots A$ & $D-\mathrm{H}$ & $\mathrm{H} \cdots A$ & $D \cdots A$ & $D-\mathrm{H} \cdots A$ \\
\hline $\mathrm{N} 1-\mathrm{H} 1 N \cdots \mathrm{O} 3$ & $0.89(2)$ & $2.19(2)$ & $2.842(2)$ & $130(2)$ \\
$\mathrm{C} 6-\mathrm{H} 6 \cdots \mathrm{O}^{\mathrm{i}}$ & 0.95 & 2.50 & $3.272(2)$ & 139 \\
$\mathrm{C} 15-\mathrm{H} 15 \cdots \mathrm{O} 2^{\mathrm{ii}}$ & 0.95 & 2.46 & $3.286(2)$ & 146 \\
$\mathrm{C} 19-\mathrm{H} 19 \cdots C g 1^{\mathrm{i}}$ & 0.95 & 2.60 & $3.407(2)$ & 143 \\
\hline
\end{tabular}

Symmetry codes: (i) $-x+1,-y+1,-z+1$; (ii) $-x+1 / 2$, $y-1 / 2, z ; C g 1$ is the centroid of the $\mathrm{C} 5 \sim \mathrm{C} 10$ ring. 


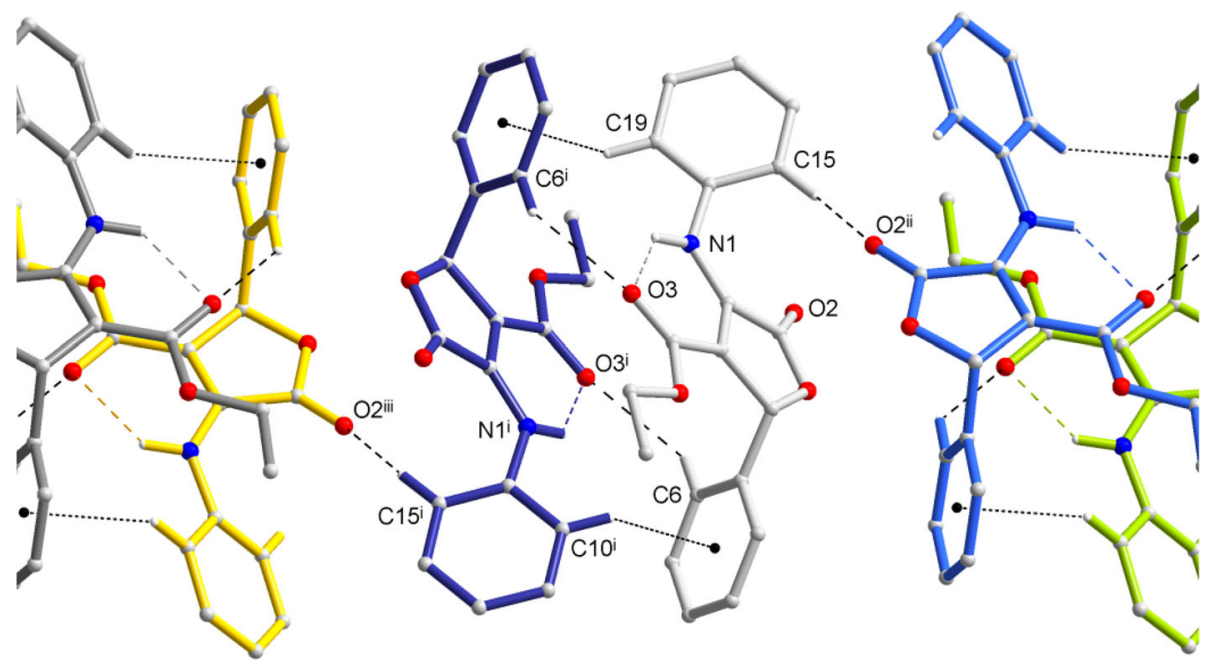

Figure 7. The arrangement of the molecules of $\mathbf{4 a}$ in the crystal lattice: centrosymmetric molecular dimers built up from two molecules (e.g., indigo-grey) joined by two $\mathrm{C}-\mathrm{H} \cdots \mathrm{O}$ and two $\mathrm{C}-\mathrm{H} \cdots \pi$ interactions (dashed and dotted lines, respectively), and the inter-dimeric $\mathrm{C} 15-\mathrm{H} 15 \cdots \mathrm{O} 2^{\mathrm{ii}}$ contacts. Symmetry codes are given in Table 5; (iii) $x+0.5,-y+1.5,-z+1$.

for the synthesis of different furan-2(5H)-ones. The structures of all products were concluded by FT-IR, ${ }^{1} \mathrm{H}$ NMR and ${ }^{13} \mathrm{C}$ NMR spectral data. The structure of compound 4a was additionally confirmed by singlecrystal X-ray diffraction. The compound crystallizes in the centrosymmetric space group Pbca. Thus, the crystal contains racemic compound $4 \mathbf{a}$. The molecular structure of $(R)$-enantiomer is presented in Figure 6.

The $\mathrm{C}(\mathrm{O}) \mathrm{OEt}$ group is coplanar with the plane of the furan-2 $(5 H)$-one ring, which is reflected by the values of the following torsion angles that are close to $0^{\circ}$ or $180^{\circ}$ : $\left[\mathrm{C} 11-\mathrm{O} 4-\mathrm{C} 12-\mathrm{C} 13179.44(10)^{\circ}\right.$; $\mathrm{C} 12-\mathrm{O} 4-\mathrm{C} 11-\mathrm{O} 33.20(16)^{\circ}$; $\mathrm{C} 2-\mathrm{C} 3-\mathrm{C} 11-$ $\mathrm{O} 33.78(18)^{\circ}$; $\left.\mathrm{N} 1-\mathrm{C} 2-\mathrm{C} 3-\mathrm{C} 11-3.46(19)^{\circ}\right]$. The $\mathrm{C}=\mathrm{O}_{\text {ester }}, \mathrm{C}-\mathrm{NH}$ syn-periplanar arrangement is stabilized by the intramolecular $\mathrm{N} 1-\mathrm{H} 1 N \cdots \mathrm{O} 3$ hydrogen bond $(\mathrm{H} \cdots \mathrm{O} 2.19(2) \AA, \mathrm{N} \cdots \mathrm{O} 2.842(2) \AA, \mathrm{N}-\mathrm{H} \cdots \mathrm{O}$ angle $\left.130(2)^{\circ}\right)$. The $\mathrm{N}$-bonded phenyl ring is slightly displaced out of the furan $2(5 \mathrm{H})$-one plane [C14-N1$\left.\mathrm{C} 2-\mathrm{C} 1-29.06(17)^{\circ}\right]$, and is additionally twisted relative to the N1-C2 bond [C2 - N1 - C14 - C15 $\left.24.35(17)^{\circ}\right]$. Thus, the angle between the $\mathrm{Ph}$ ring and furan $2(5 H)$-one moiety is about $45^{\circ}$. The geometrical parameters for $\mathbf{4 a}$ are given in the crystallographic information file (CIF) provided as Supplementary Information.

In the crystal lattice of $\mathbf{4 a}$, two molecules of the opposite chirality are joined to each other by two $\mathrm{C}-\mathrm{H} \cdots \mathrm{O}$ and two $\mathrm{C}-\mathrm{H} \cdots \pi$ interactions (Table 5). The adjacent dimers are joined to each other by the $\mathrm{C} 15-\mathrm{H} 15 \cdots \mathrm{O} 2^{\mathrm{ii}}$ contacts to form tapes displayed in Figure 7.

\section{Conclusions}

One-pot three-component synthesis of a variety of furanone derivatives has been developed using tetramethylguanidine-functionalized silica-coated iron oxide magnetic nanoparticles $\left(\mathrm{Fe}_{3} \mathrm{O}_{4}\right.$-TMG) as a green, efficient and recyclable catalyst. The mild reaction conditions, simple work-up, the good-to-high yield of products and use of magnetic nanocatalyst are some of the superior features of this method.

\section{Supplementary Information (SI)}

Copies of the original spectra, FT-IR (Figures S1, S4, S7, S10, S13, S16, S19, S22, S25, S28, S31, S34, S37, S40, S43), ${ }^{1} \mathrm{H}$ NMR (Figures S2, S5, S8, S11, S14, S17, S20, S23, S26, S29, S32, S35, S38, S41, S44), ${ }^{13}$ C NMR (S3, S6, S9, S12, S15, S18, S21, S24, S27, S30, S33, S36, S39, S42, S45) of all the compound reported in the experimental section, TGA (Figures S46 and S47) and FT-IR spectrum of tetramethylguanidine (Figure S48) and crystallographic information of compound 4a (CIF file) provided are included in Supplementary Information. Supplementary Information is available at www.ias.ac.in/chemsci.

\section{Acknowledgements}

This work is funded by the grant NRF-2018R1A2B3001246 of the National Research Foundation of Korea.

\section{References}

1. Eften'eva R I, Kushnir O V, Lyavinets O S, Mangalagiu I I and Vovk M V 2016 Synthesis of polyfunctionalized pyrido $[1,2-\mathrm{a}]$ pyrazines and pyrazino $[1,2-\mathrm{a}]$ 
quinolines via one-pot multicomponent reactions Monatsh. Chem. 1472127

2. Ahankar H, Ramazani A, Ślepokura K, Lis T and Joo S W 2016 Synthesis of pyrrolidinone derivatives from aniline, an aldehyde and diethyl acetylenedicarboxylate in an ethanolic citric acid solution under ultrasound irradiation Green Chem. 183582

3. Ramazani A, Nasrabadi F Z, Rezaei A, Rouhani M, Ahankar H, Asiabi P A, Joo S W, Ślepokura K and Lis T 2015 Synthesis of $\mathrm{N}$-acylurea derivatives from carboxylic acids and $\mathrm{N}, \mathrm{N}^{\prime}$-dialkyl carbodiimides in water J. Chem. Sci. 1272269

4. Prakash S, Jayamoorthy K, Srinivasan N, Rajasekar T and Subash B $2017 \mathrm{Nano}_{-} \mathrm{SiO}_{2}$ : An efficient catalyst for the synthesis of some new benzimidazoles-NMR spectral characterization Silicon 9949

5. Ahankar H, Ramazani A and Joo S W 2016 Magnetic nickel ferrite nanoparticles as an efficient catalyst for the preparation of polyhydroquinoline derivatives under microwave irradiation in solvent-free conditions Res. Chem. Intermed. 422487

6. Fardood S T, Ramazani A and Moradi S 2017 Green synthesis of $\mathrm{Ni}-\mathrm{Cu}-\mathrm{Mg}$ ferrite nanoparticles using tragacanth gum and their use as an efficient catalyst for the synthesis of polyhydroquinoline derivatives J. Sol. Gel. Sci. Technol. 82432

7. Ahankar H, Ramazani A, Slepokura K, Lis T and Joo S W 2018 One-pot synthesis of substituted 4H-chromenes by nickel ferrite nanoparticles as an efficient and magnetically reusable catalyst Turk. J. Chem. 42719

8. Nazari S, Saadat S, Fard P K, Gorjizadeh M, Nezhad E R and Afshari M 2013 Imidazole functionalized magnetic $\mathrm{Fe}_{3} \mathrm{O}_{4}$ nanoparticles as a novel heterogeneous and efficient catalyst for synthesis of dihydropyrimidinones by Biginelli reaction Monatsh. Chem. 1441877

9. Ghavami M, Koohi M and Kassaee MZ 2013 A selective nanocatalyst for an efficient Ugi reaction: Magnetically recoverable $\mathrm{Cu}(\mathrm{acac})_{2} / \mathrm{NH}_{2}-\mathrm{T} / \mathrm{SiO}_{2} @ \mathrm{Fe}_{3} \mathrm{O}_{4}$ nanoparticles J. Chem. Sci. 1251347

10. Ahmadi E, Ramazani A, Hamdi Z, MashhadiMalekzadeh A and Mohamadnia Z 2015 5, 10, 15, 20tetrakis (4-carboxyphenyl) porphyrin covalently bound to nano-silica surface: preparation, characterization and chemosensor application to detect TNT Silicon 7323

11. Ladol J, Khajuria H, Sheikh H N and Khajuria Y 2016 Synthesis and characterization of bi-functional magnetoluminescent $\mathrm{Fe}_{3} \mathrm{O}_{4} @ \mathrm{SiO}_{2} @ \mathrm{NaLuF}_{4}$ : $\mathrm{Eu}^{3+}$ hybrid core/shell nanospheres J. Chem. Sci. 1281149

12. Dömling A and Ugi I 2000 Multicomponent reactions with isocyanides Angew. Chem. Int. Ed. 393168

13. Orru R V and de Greef M 2003 Recent advances in solution-phase multicomponent methodology for the synthesis of heterocyclic compounds Synthesis 101471

14. Zhu J 2003 Recent developments in the isonitrile-based multicomponent synthesis of heterocycles Eur. J. Org. Chem. 20031133

15. Ramón D J and Yus M 2005 Asymmetric multicomponent reactions (AMCRs): the new frontier Angew. Chem. Int. Ed. 441602

16. Hashem A I, Senning A and Hamad A-S S 1998 Photochemical transformations of $2(5 \mathrm{H})$-furanones. A review Org. Prep. Proced. Int. 30401
17. Weber V, Coudert P, Rubat C, Duroux E, Vallée-Goyet D, Gardette D, Bria M, Albuisson E, Leal F and Gramain J-C 2002 Novel 4,5-diaryl-3-hydroxy-2 (5H)-furanones as anti-oxidants and anti-inflammatory agents Bioorg. Med. Chem. 101647

18. Brown S P, Goodwin N C and MacMillan D W 2003 The first enantioselective organocatalytic MukaiyamaMichael reaction: a direct method for the synthesis of enantioenriched $\gamma$-butenolide architecture J. Am. Chem. Soc. 1251192

19. Roethle P A and Trauner D 2006 Expedient synthesis of ( \pm -bipinnatin j Org. Lett. 8345

20. San Feliciano A, Barrero A, Medarde M, del Corral J M, Aizpiri A A and Sánchez-Ferrando F 1984 Asteriscunolides A, B, C and D, the first humulanolides; Two pairs of conformationally stable stereoisomers Tetrahedron $\mathbf{4 0} 873$

21. Seki T, Satake M, Mackenzie L, Kaspar H F and Yasumoto T 1995 Gymnodimine, a new marine toxin of unprecedented structure isolated from New Zealand oysters and the dinoflagellate, Gymnodinium sp. Tetrahedron Lett. 367093

22. Murakami T, Sasaki A, Fukushi E, Kawabata J, Hashimoto M and Okuno T 2005 Lambertellol C, a labile and novel biosynthetic congener of lambertellols A and B Bioorg. Med. Chem. Lett. 152587

23. Reddy N S, Venkatesham U, Rao T P and Venkateswarlu Y 2000 New sesquiterpenes from the marine sponge Dysidea fragilis Indian J. Chem. 393

24. Takahashi S, Kubota A and Nakata T 2002 Total synthesis of muconin Tetrahedron Lett. 438661

25. Hanessian S, Park H and Yang R-Y 1997 Zinc-mediated allylation of $n$-protected $\alpha$-amino aldehydes in aqueous solution. stereoselective synthesis of anti-and syn- $\beta$ amino alcohols with functionalized allyl groups Synlett 1997353

26. Choudhury A, Jin F, Wang D, Wang Z, Xu G, Nguyen D, Castoro J, Pierce M E and Confalone P N 2003 A concise synthesis of anti-viral agent $\mathrm{F}$-ddA, starting from (S)-dihydro-5-(hydroxymethyl)-2 (3H)-furanone Tetrahedron Lett. 44247

27. Grossmann G, Poncioni M, Bornand M, Jolivet B t, Neuburger M and Séquin U 2003 Bioactive butenolides from Streptomyces antibioticus TÜ 99: absolute configurations and synthesis of analogs Tetrahedron 59 3237

28. Hein S M, Gloer J B, Koster B and Malloch D 2001 Bombardolides: new antifungal and antibacterial $\gamma$-Lactones from the coprophilous fungus bombardioidea anartia $J$. Nat. Prod. 64809

29. Padakanti S, Pal M and Yeleswarapu K R 2003 An improved and practical synthesis of 5,5dimethyl-3-(2-propoxy)-4-(4-methanesulfonylphenyl)2- $(5 \mathrm{H})$-furanone (DFP-a selective inhibitor of cyclooxygenase-2) Tetrahedron 597915

30. Murthy S N, Madhav B, Kumar A V, Rao K R and Nageswar Y 2009 Facile and efficient synthesis of 3,4,5substituted furan-2(5H)-ones by using $\beta$-cyclodextrin as reusable catalyst Tetrahedron $\mathbf{6 5} 5251$

31. Doostmohammadi R, Maghsoodlou M T, Hazeri N and Habibi-Khorassani S M 2013 An efficient one-pot multi-component synthesis of 3,4,5-substituted furan-2 
$(5 \mathrm{H})$-ones catalyzed by tetra- $n$-butylammonium bisulfate Chin. Chem. Lett. 24901

32. Shafiee M R M, Mansoor S S, Ghashang M and Fazlinia A 2014 Preparation of 3,4,5-substituted furan-2 (5H)ones using aluminum hydrogen sulfate as an efficient catalyst C. R. Chim. 17131

33. Nagarapu L, Kumar U N, Upendra P and Bantu R 2012 Simple, convenient method for the synthesis of substituted furan-2 (5H)-one derivatives using tin (II) chloride Synth. Commun. 422139

34. Hazeri N, Taher Maghsoodlou M, Mahmoudabadi N, Doostmohammadi R and Salahi S 2014 Sucrose as an environmental and economical catalyst for the synthesis of $2(5 \mathrm{H})$ furanone Curr. Organocatal. 145

35. Kangani M, Maghsoodlou M-T and Hazeri N 2016 Vitamin B12: An efficient type catalyst for the onepot synthesis of 3,4,5-trisubstituted furan-2 $(5 \mathrm{H})$-ones and N-aryl-3-aminodihydropyrrol-2-one-4-carboxylates Chin. Chem. Lett. 2766

36. Basyouni W, El-Bayouki K A, El-Sayed A, Tohamy W, Farag M and Abd-El-Baseer M 2015 3,4,5-Trisubstituted furan-2 $(5 \mathrm{H})$-one derivatives: Efficient one-pot synthesis and evaluation of cytotoxic activity Drug Res. 65473

37. Doostmohammadi R and Hazeri N 2013 Application of silica gel-supported polyphosphoric acid (PPA/ $\mathrm{SiO}_{2}$ ) as a reusable solid acid catalyst for one-pot multicomponent synthesis of 3,4,5-substituted furan-2 $(5 \mathrm{H})$ ones Lett. Org. Chem. 10199

38. Sajadikhah S S and Zarei M 2017 Green and efficient synthesis of functionalized 3-amino-2-oxofuranes using trityl chloride Iran. Chem. Commun. 5436

39. Doostmohammadi R, Maghsoodlou M T, Hazeri N and Habibi-Khorassani S M 2013 Acetic acid as an efficient catalyst for the one-pot preparation of 3,4,5-substituted furan-2 (5H)-ones Res. Chem. Intermed. 394061

40. Doostmohammadi R, Maghsoodlou M T and HabibiKhorassani S M 2012 Formic acid as an efficient catalyst for the one-pot preparation of furan-2 $(5 \mathrm{H})$-ones under solvent-free condition Iran J. Org. Chem. 4939

41. Ramesh S and Nagarajan R 2011 Efficient one-pot multicomponent synthesis of (carbazolylamino) furan-2 (5H)-one and carbazolyltetrahydropyrimidine derivatives Synthesis 20113307

42. Salahi S, Maghsoodlou M T and Hazeri N 2015 Brosted acidic ionic liquid ([Bmim] $\mathrm{HSO}_{4}$ ) promoted cyclocodensation reaction: Synthesis of 3,4,5-substituted furan2 (5H)-ones Orient. J. Chem. 312047

43. Salahi S, Maghsoodlou M T, Hazeri N, Movahedifar F, Doostmohammadi R and Lashkari M 2015 Acidic ionic liquid N-methyl 2-pyrrolidonium hydrogen sulfate as an efficient catalyst for the one-pot multicomponent preparation of 3,4,5-substituted furan-2 (5H)-ones Res. Chem. Intermed. 416477
44. Adrom B, Maghsoodlou M T, Lashkari M, Hazeri N and Doostmohammadi R 2016 Efficient one-pot threecomponent synthesis of 3,4,5-substituted furan-2 (5 $\mathrm{H})$-ones catalyzed watermelon juice Synth. React. Inorg. Met. Org. Chem. 46423

45. Hazeri N, Doostmohammadi R, Adrom B, Lashkari M and Maghsoodlou M T 2016 Extract of barberry as entirely green catalyst for the synthesis of structurally diverse 3,4,5-substituted furan-2 (5H)-ones Chem. J. Mold. 1168

46. Khan M M, Khan S and Sahoo S C 2018 Efficient and eco-friendly one-pot synthesis of functionalized furan2-one, pyrrol-2-one, and tetrahydropyridine using lemon juice as a biodegradable catalyst ChemistrySelect $\mathbf{3}$ 1371

47. Bahramian F, Fazlinia A, Mansoor S S, Ghashang M, Azimi F and Biregan M N 2016 Preparation of 3, 4, 5-substituted furan-2 (5H)-ones using HY Zeolite nanopowder as an efficient catalyst Res. Chem. Intermed. 42 6501

48. Tekale S U, Kauthale S S, Pagore V P, Jadhav V B and Pawar R P 2013 ZnO nanoparticle-catalyzed efficient one-pot three-component synthesis of 3,4,5trisubstituted furan-2 (5H)-ones J. Iran. Chem. Soc. 10 1271

49. Asghari S and Mohammadnia M 2017 Preparation and characterization of sulfamic acid pyridinium chloridefunctionalized $\mathrm{Fe}_{3} \mathrm{O}_{4}$ nanoparticles as a novel magnetic catalyst for synthesis of novel $N$-coumarin-2-furanones Res. Chem. Intermed. $\mathbf{4 3} 7193$

50. Saha M and Das A R 2017 Access of diverse 2pyrrolidinone, 3,4,5-substituted furanone and 2-oxodihydropyrroles applying graphene oxide nanosheet: Unraveling of solvent selectivity ChemistrySelect 2 10249

51. Safaei-Ghomi J, Hatami A and Shahbazi-Alavi H 2017 A highly flexible green synthesis of 3,4,5-substituted furan$2(5 \mathrm{H})$-ones using nano- $\mathrm{CdZr}_{4}\left(\mathrm{PO}_{4}\right)_{6}$ as catalyst under microwave irradiation Polycycl. Aromat. Compd. 37407

52. Shahraki M, Habibi-Khorassani S M and Dehdab $\mathrm{M}$ 2015 Effect of different substituents on the one-pot formation of 3,4,5-substituted furan-2 $(5 \mathrm{H})$-ones: a kinetics and mechanism study RSC Adv. 552508

53. Gein V, Mihalev V, Kasimova N, Voronina E, Vakhrin M and Babushkina E 2007 Synthesis and antibacterial activity of 1-alkoxyalkyl-5-aryl-4-acyl-3-hydroxy3-pyrrolin-2-ones Pharm. Chem. J. 41208

54. Sheldrick G M 2008 A short history of SHELX Acta Crystallogr. Sect. A Found. Crystallogr. 64112

55. Sheldrick G M 2015 Crystal structure refinement with SHELXL Acta Crystallogr., Sect. C Struct. Chem. 713

56. Brandenburg K 2014 DIAMOND Version 3.2k. Crystal Impact GbR: Bonn, Germany 